\title{
NubiVis: A Personal Cloud File Explorer
}

\author{
Josef Spillner \\ Technische Universität \\ Dresden \\ Faculty of Computer Science \\ Cloud Storage Lab \\ 01062 Dresden, Germany \\ josef.spillner@tu- \\ dresden.de
}

\author{
Sebastian Tilsch \\ Technische Universität \\ Dresden \\ Faculty of Computer Science \\ Cloud Storage Lab \\ 01062 Dresden, Germany \\ sebastian.tilsch@mailbox.tu- \\ dresden.de
}

\author{
Alexander Schill \\ Technische Universität \\ Dresden \\ Faculty of Computer Science \\ Chair for Computer Networks \\ 01062 Dresden, Germany \\ alexander.schill@tu- \\ dresden.de
}

\begin{abstract}
We introduce and demonstrate NubiVis, a web-based tool for exploring and managing personal files stored in arbitrary locations with a unified view. NubiVis supports views on files partitioned and dispersed across several storage services when running atop the NubiSave cloud storage controller, as well as meta-data and facets when run in combination with the Strigi file indexer. In addition to the visualisation through various extensible modules, NubiVis also enables users to execute control over where their files are stored by simulated and actual exclusion of storage providers. No server-side infrastructure is required for NubiVis which makes it a suitable integrated component of mobile devices for fully user-controlled pervasive access to files.
\end{abstract}

\section{PERSONAL FILE MANAGEMENT TO- DAY}

File management has been an everlasting task for users since the beginnings of personal and desktop computers. With the introduction of peripheral devices, removeable media and online storage, an expanded view on storage management has been made a concern of these users. On recent mobile devices, which are characterised by an isolation of perapplication file stores, file and storage management plays a lesser but still important role during the daily use, in particular for synchronisation between multiple devices. Today's integrated and add-on file managers (listed in Table 1), while offering many convenient innovations such as remote folder integration and context actions on files, are not empowering users to relate their files from a unified view across providers to the logical and physical storage locations and their characteristics and associated benefits and risks. This leads to a reduced overview of which files are stored where and how and to reduced control about how they can be retrieved and managed, thus resulting in a higher dependency on the storage service providers. We call the missing concepts storage service awareness and distribution awareness, respectively.

Permission to make digital or hard copies of all or part of this work for personal or classroom use is granted without fee provided that copies are not made or distributed for profit or commercial advantage and that copies bear this notice and the full citation on the first page. To copy otherwise, to republish, to post on servers or to redistribute to lists, requires prior specific permission and/or a fee.

MOBIQUITOUS 2014, December 02-05, London, Great Britain

Copyright (c) 2014 ICST 978-1-63190-039-6

DOI 10.4108/icst.mobiquitous.2014.258064
Table 1: Selection of integrated and freely available file managers

\begin{tabular}{|l|l|l|}
\hline File manager & Operating system & S/D Awareness \\
\hline \hline Explorer & Microsoft Windows & - \\
\hline Finder & Apple MacOS X & - \\
\hline Dolphin & KDE & - \\
\hline Nautilus & GNOME & - \\
\hline Files & Microsoft Windows Phone & - \\
\hline Astro & Android (add-on) & - \\
\hline Forklift & Apple MacOS X (add-on) & - \\
\hline iStorage & Apple iOS (add-on) & - \\
\hline
\end{tabular}

Previously, researchers have explored advanced visualisation and interaction concepts for collaborative file management [6] and semantic navigation hierarchies [2]. To the best of our knowledge, there is no focused attempt yet at file management concepts for distributed cloud environments which uses such concepts for increased user awareness and corresponding control facilities.

\section{MANAGEMENT OF PERVASIVE DATA ACROSS CLOUDS}

Pervasive access to personal data across devices is a key argument for online file storage. Several cloud storage controllers have been proposed and implemented $[7,3]$. These controllers combine several storage services and thus raise the availability above a level which could be achieved by single services, while at the same time increasing security and limiting capacity overhead.

However, the controllers and their dispersal schemes are not sufficient in practice. Users and data producers expect an effective control facility concerning the storage goals. In an enterprise context, they furthermore expect compliance with storage policies [5]. An effective control requires the collection, curation and visualisation of information. Similar techniques are already in use today for the detection of incidents and digital forensics [1]. Therefore, the need to extend storage controllers with visualisation and control facilities becomes evident, and the choice to integrate them into the file management paradigm already known to users is a logical corollary.

The NubiSave cloud storage controller in particular offers a filesystem interface with hot-pluggable storage service modules to let existing applications benefit from such a scheme 
[4]. Underneath, NubiSave replicates and disperses files with specified encoding and splitting parameters to multiple storage services. Due to this design, we can follow a separation of concerns approach in which the file visualisation and exploration itself is not concerned with the actual file storage management, service interfaces, service failures or synchronisation issues. Rather, the explorer's task is to let the user configure all aspects of the storage controller and to give feedback about stored files, failure statistics, and proposed actions.

\section{THE NUBIVIS APPROACH}

We designed and built a web-based file manager which can run both on intermediary servers for multi-user and crossdevice setups and directly on the end device for full control by the user. The file manager is called NubiVis which, as the name suggests, focuses on the visualisation of files which are stored in a distributed manner. However, on top of the visualisation, it contains several feedback and control channels. The component architecture of NubiVis is shown in Fig. 1. It consists of a web interface and a local or remote web service to which it is connected. The service reads the file system information and enriches it with semantic and cloud distribution meta-data by accessing the relevant databases of Strigi, a file indexing tool, and NubiSave, the cloud storage controller, respectively. The NubiSave database contains the provider and fragment distribution information as well as information about the state and the general non-functional properties of the storage services. The architecture allows for the addition of further frontends, including native mobile applications and enhanced native file managers through plugins.

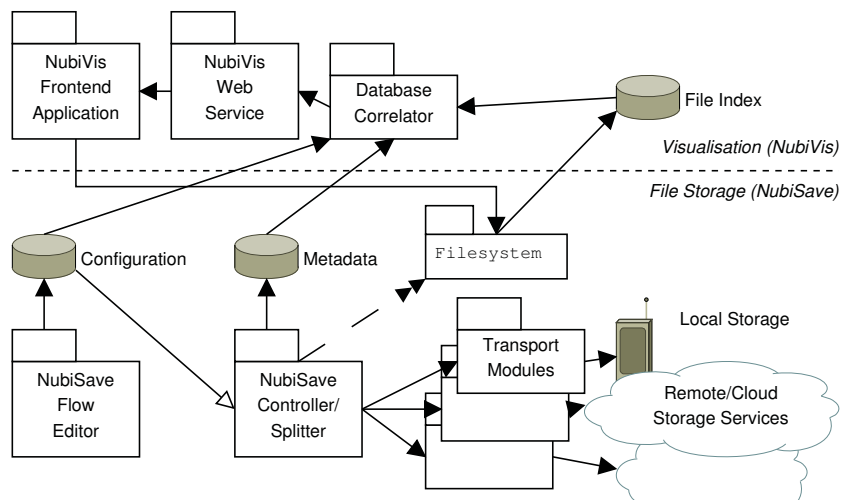

Figure 1: Architecture of NubiVis

NubiVis is designed to be highly modular. While the faceted and full-text search as well as the filtering are centrally available navigation elements, the settings and administration as well as the visualisation and control views can be added and removed as needed. Currently, the following views exist.

- Home: Home screen with overview statistics.

- Map: Geographic distribution of either physical file locations, i.e. provider data centre coordinates, or file origins, such as photo coordinates.
- Globe: Similar to map, a three-dimensional file locator.

- Tree: Traditional tree view, enhanced with file distribution and redundancy information.

- Bubbles: Simple bubble view over all files.

- Size Chart: Hierarchical directory and file size proportions.

- Storages: Distribution-centric view of all services and their properties, including capacity.

- Discover: File preview browser.

- Documents: Documents view.

- Distribution: Configuration of the file distribution policies.

- Timeline: Chronologic view of when which files were stored or changed.

The filter feature allows user to exclude certain storage providers. The view dynamically adapts to this setting and hence simulates the unavailability of the selected provider. Experienced users can verify their dependency on certain providers. NubiVis hence answers the typical question of Where is my data? in an increasingly mobile and distributed personal data world.

\section{IMPLEMENTATION AND DEMO SCOPE}

The NubiVis frontend is implemented as a web application with JavaScript and the Dojo framework. Its RESTful web service backend is implemented as a Java servlet application. The integrated system will be demonstrated on a notebook or tablet PC with several scenario files which are to be stored in the cloud. Each cloud provider is emulated on the device itself with a local storage service (WebDAV, SSH or CIFS). A sudden unavailability of providers can be provoked at any time.

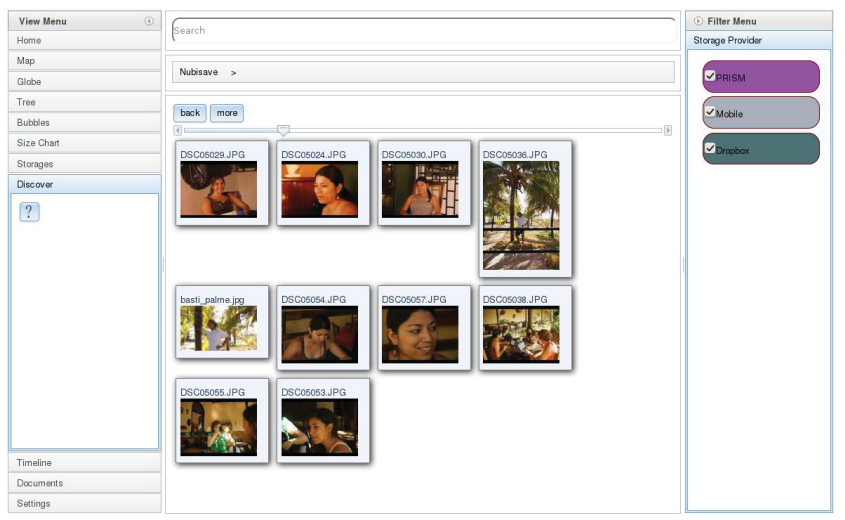

Figure 2: Modular views aiding the user in the discovery and exploration of personal files

The general appearance of the explorer is conveyed in the screenshots in Fig. 2 and Fig. 3a-c. The user is able to use a unified interface, and yet when necessary take a look at 

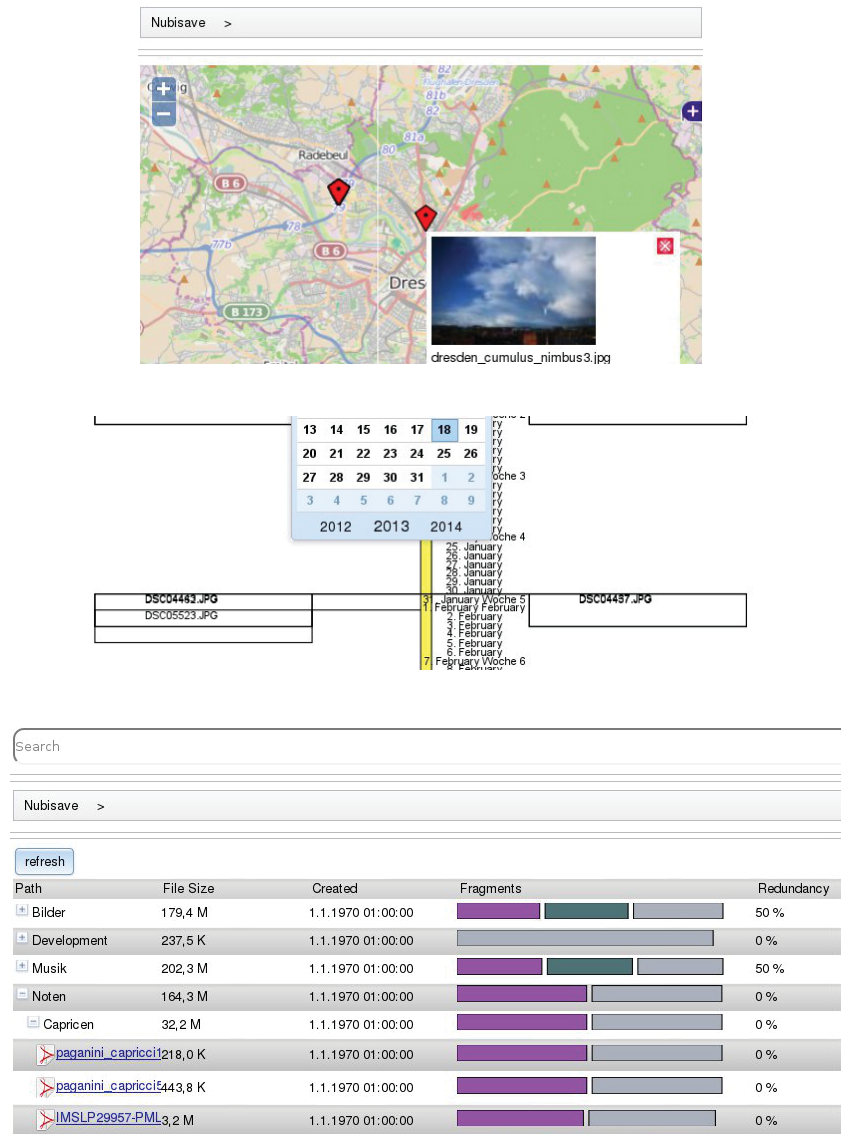

Figure 3: Examples for views: (a) map; (b) timeline; (c) tree view with fragment information

where and how the files and, when running with a dispersing storage controller, the fragments are distributed.

Fig. 4 shows a screenshot of one particular module, Distribution, which enables users to view and change their fragment distribution. Changes are performed by selecting a fragment and performing a migration decision. Fig. 5 shows the resulting migration dialogue. Upon execution, the file is moved and the meta-data about the distributed is updated by the storage controller.

NubiVis is free software available for download along with documentation, screenshots and a screencast at its dedicated website ${ }^{1}$.

\section{ACKNOWLEDGEMENTS}

This work has been partially funded by the German Research Foundation (DFG) under project agreements SCHI 402/11-1.

\section{REFERENCES}

[1] S. Axelsson. Visualisation for Intrusion Detection Hooking the Worm. In proceedings of the 8th European Symposium on Research in Computer Security

\footnotetext{
${ }^{1}$ NubiVis website: http://nubisave.org/nubivis/
}

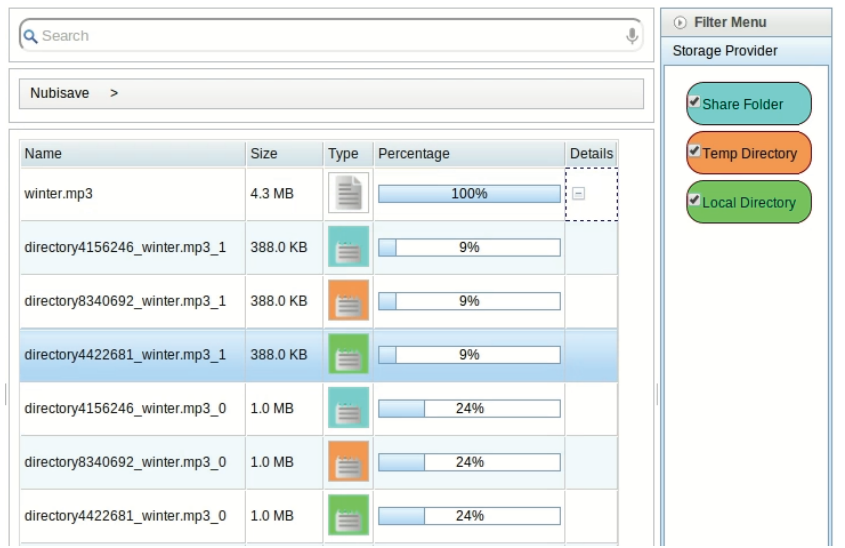

Figure 4: Fragment browser used for migration decisions

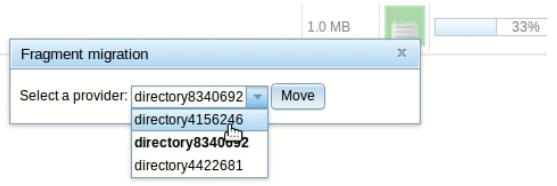

Figure 5: Dialogue to perform a fragment migration

(ESORICS), volume 2808 of Lecture Notes in Computer Science (LNCS), pages 101-117, 2003.

[2] P. T. Daniel and G. M. J. Fonseca. Interactive File Searching Using File Metadata and Visualization. In Proceedings of the 24th BCS Interaction Specialist Group Conference (BCS), pages 27-35, Dundee, Scotland, United Kingdom, September 2010.

[3] R. M. D. O. Libardi, M. V. N. Bedo, S. Reiff-Marganiec, and J. C. Estrella. MSSF: A Step towards User-Friendly Multi-Cloud Data Dispersal. In 7th IEEE International Conference on Cloud Computing (CLOUD), pages 952-953, Anchorage, Alaska, USA, June 2014.

[4] J. Spillner, J. Müller, and A. Schill. Creating Optimal Cloud Storage Systems. Future Generation Computer Systems, 29(4):1062-1072, June 2013. DOI: http://dx.doi.org/10.1016/j.future.2012.06.004.

[5] J. Spillner and A. Schill. Flexible Data Distribution Policy Language and Gateway Architecture. In 1st Latin American Conference on Cloud Computing and Communications (LatinCloud), pages 1-6, Porto Alegre, Brazil, November 2012.

[6] T. Whalen, E. G. Toms, and J. Blustein. Information Displays for Managing Shared Files. In Proceedings of the 2nd ACM Symposium on Computer Human Interaction for Management of Information Technology (CHIMIT), San Diego, California, USA, November 2008.

[7] H.-S. Yeo, X.-S. Phang, H.-J. Lee, and H. Lim. Leveraging client-side storage techniques for enhanced use of multiple consumer cloud storage services on resource-constrained mobile devices. Journal of Network and Computer Applications, 43(1):142-156, August 2014. DOI: 10.1016/j.jnca.2014.04.006. 